\title{
Dynamic shop floor re-scheduling approach inspired by a neuroendocrine regulation mechanism
}

Proc IMechE Part B:

$J$ Engineering Manufacture

20I5, Vol. 229(SI) I2I-134

(C) IMechE 2015

Reprints and permissions:

sagepub.co.uk/journalsPermissions.nav DOI: I0.1 I 77/0954405414558699

pib.sagepub.com

@SAGE

\author{
Kun Zheng', Dunbing Tang', Adriana Giret ${ }^{2}$, Wenbin Gu ${ }^{1,3}$ and Xing Wu'
}

\begin{abstract}
With the development of the market globalisation trend and increasing customer orientation, many uncertainties have entered into the manufacturing context. To create an agile response to the emergence of and change in conditions, this article presents a dynamic shop floor re-scheduling approach inspired by a neuroendocrine regulation mechanism. The dynamic re-scheduling function is the result of cooperation among several autonomous bio-inspired manufacturing cells with computing power and optimisation capabilities. The dynamic re-scheduling model is designed based on hormone regulation principles to agilely respond to the frequent occurrence of unexpected disturbances at the shop floor level. The cooperation mechanisms of the dynamic re-scheduling model are described in detail, and a test bed is set up to simulate and verify the dynamic re-scheduling approach. The results verify that the proposed method is able to improve the performances and enhance the stability of a manufacturing system.
\end{abstract}

\section{Keywords}

Dynamic re-scheduling, neuroendocrine-inspired manufacturing system, bio-inspired manufacturing cell, neuroendocrine regulation, hormone regulation

Date received: 8 July 20|4; accepted: 13 October 2014

\section{Introduction}

With the development of the market globalisation trend and increasing customer orientation in manufacturing enterprises, increasingly personalised and diversified demands bring different types of uncertainties into the manufacturing system, that is, dynamic task change, rush orders, and manufacturing equipment failure. To meet these challenges, the control structure of contemporary manufacturing systems is becoming additionally complex, and the requirements for agility and robustness are also increasing. Therefore, the contemporary manufacturing system requires a good dynamic scheduling mechanism to rapidly respond to uncertainties and improve production efficiency by optimising the allocation of tasks and manufacturing resources under certain manufacturing constraints (product processing costs, resource utilisation and delivery time, etc.). ${ }^{1}$

Recently, numerous researchers have focused on further studies of manufacturing dynamic scheduling and have produced many achievements. The results show that resolution of dynamic scheduling is primarily realised by means of meta-heuristic and multi-agent technology. Yandra and Hiroyuki $^{2}$ applied a genetic algorithm featuring heterogeneous population to solve multi-objective flow shop scheduling problems. Fattahi and Fallahi $^{3}$ presented a mathematical model for a dynamic and flexible job shop scheduling approach and used a genetic algorithm to improve the efficiency and stability of schedules. Rakesh et al. $^{4}$ used a simulation-based genetic algorithm optimisation method and heuristics to solve process-planning and scheduling problems. In general, a good solution can be obtained using meta-heuristic algorithms, but the required computing time and implementation effort still pose limitations to these approaches. Agent-based

\footnotetext{
'College of Mechanical and Electrical Engineering, Nanjing University of Aeronautics and Astronautics, Nanjing, China

${ }^{2}$ Departamento de Sistemas Informáticos y Computación, Universidad Politécnica de Valencia, Valencia, Spain

${ }^{3}$ College of Mechanical and Electrical Engineering, Hohai University,

Changzhou Campus, Changzhou, China
}

\section{Corresponding author:}

Dunbing Tang, College of Mechanical and Electrical Engineering, Nanjing University of Aeronautics and Astronautics, 29 Yudao Street, Nanjing 210016, China.

Email: d.tang@nuaa.edu.cn 
technology offers advantages for distributed scheduling, including improved reaction to disturbances and parallel computation. Renna ${ }^{5}$ proposed a coordination approach for multi-agent architecture based on computation of internal and external indices of the generic manufacturing cell. Qin and $\mathrm{Kan}^{6}$ introduced a new production dynamic scheduling method based on an improved contract net of multi-agents used to solve the dynamic and complicated problem of production scheduling. Iwamura et al. ${ }^{7}$ applied a real-time scheduling approach based on utility values to holonic manufacturing systems. Jana et al. ${ }^{8}$ applied a Contract Net Protocol (CNP) to address negotiation-based task allocation of resources for preparation of dynamic scheduling in an agent-based holonic control framework. The negotiation mechanism based on CNP has the advantages of a simple strategy and convenient application, but it is a type of explicit coordination mechanism. Due to the increasing complication and dynamics of the manufacturing environment, the limitation of the CNP coordination mechanism is its enormous need for communications, which has a tendency to appear in the process of coordination, negotiation, and cooperation. ${ }^{9}$

With expansion and further research in artificial intelligence, intelligent modelling of the human body's information processing mechanism has become a new research focus in recent years. The diversity, complexity, reliability, and adaptability of the system structure, function, and regulatory mechanisms are worthy of reference in studying manufacturing systems. The neuroendocrine system is a core component of the human body's information processing mechanism, and this complex and unique information processing mechanism gives researchers much inspiration. A coordination methodology based on the neuroendocrine regulation mechanism is one type of implicit coordination approach that leads to rapid coordination between individuals as well as coordination among all system resources. Using this mechanism, the system can achieve rapid synchronisation and cooperation to achieve global optimisation. Compared with other coordination mechanisms (CNP, etc.), this mechanism offers fewer communications, simpler coordination, and easier implementation. ${ }^{10}$

Inspired by the neuroendocrine regulation mechanism, we propose a neuroendocrine-inspired manufacturing system (NEIMS) and describe the NEIMS architecture $^{11}$ in detail. Based on the NEIMS architecture, this article designs a dynamic re-scheduling approach that can agilely address different types of emergencies at the shop floor level to optimise task and resource allocations. The aim of this research is to improve the performance indicators of the manufacturing system by applying the mechanism of neuroendocrine regulation, thus consolidating and deepening the NEIMS theory and fundamentals.

The remainder of this article is organised as follows. The section 'Neuroendocrine-inspired dynamic rescheduling architecture' presents the main principles of a neuroendocrine-inspired dynamic re-scheduling architecture, that is, NEIMS components, dynamic rescheduling model, and resource allocation mechanism based on the hormone regulation mechanism. The section 'Cooperation mechanisms for dynamic re-scheduling' illustrates three types of cooperation mechanisms for dynamic re-scheduling in NEIMS that use the hormone regulation mechanism to agilely respond to disturbances and maintain the system with relative stability. The section 'Test bed for NEIMS simulation' introduces a pilot test bed for simulation of the proposed dynamic re-scheduling approach in NEIMS. The section 'Simulation study' describes comparisons with other approaches relative to the potential of improving the system performance, and the last section 'Conclusion' gives a summary of the article and an introduction to future study.

\section{Neuroendocrine-inspired dynamic re-scheduling architecture}

\section{NEIMS components}

The NEIMS control architecture (Figure 1) is built on a set of autonomous and cooperative units, referred to as the bio-inspired manufacturing cell (BIMC) (Figure 2), which represent the manufacturing components. The functional components of the BIMC are the controller, decision maker, and perception components. The perception unit of the BIMC can perceive exterior and interior environmental stimuli, which trigger the decisions required for agile reaction. After a decision is made, the controller gives a command to the relevant BIMCs to execute the decision.

A BIMC is defined as an autonomous entity that can perform certain tasks, achieve a goal autonomously, and regulate itself when facing non-pre-deterministic changes in the manufacturing environment. The design of a basic BIMC incorporates a set of pertinent attributes that can fully represent any level in the architecture hierarchy. In other words, a BIMC can represent an entire manufacturing shop at the highest level, a manufacturing cell at the middle level, or a physical machine at the bottom level. Therefore, the architecture exhibits the explicit characteristic of recursiveness.

A BIMC at the shop floor level plays the role of supervisor to provide optimisation and coordination services to the BIMCs under its supervision. This unit can handle complicated scheduling problems and elaborate the schedule plans due to the powerful computing power and optimisation capability embedded within it. Elaboration of a schedule plan may require a large amount of computing time and effort, but the plan will be optimal because of the global view of the BIMC at the shop floor level.

A BIMC at the cell level plays the role of supervisor in its own cell to provide optimisation and order execution services to the BIMCs under its supervision and coordination services to the BIMCs at all levels. This unit can address scheduling problems in its own cell 


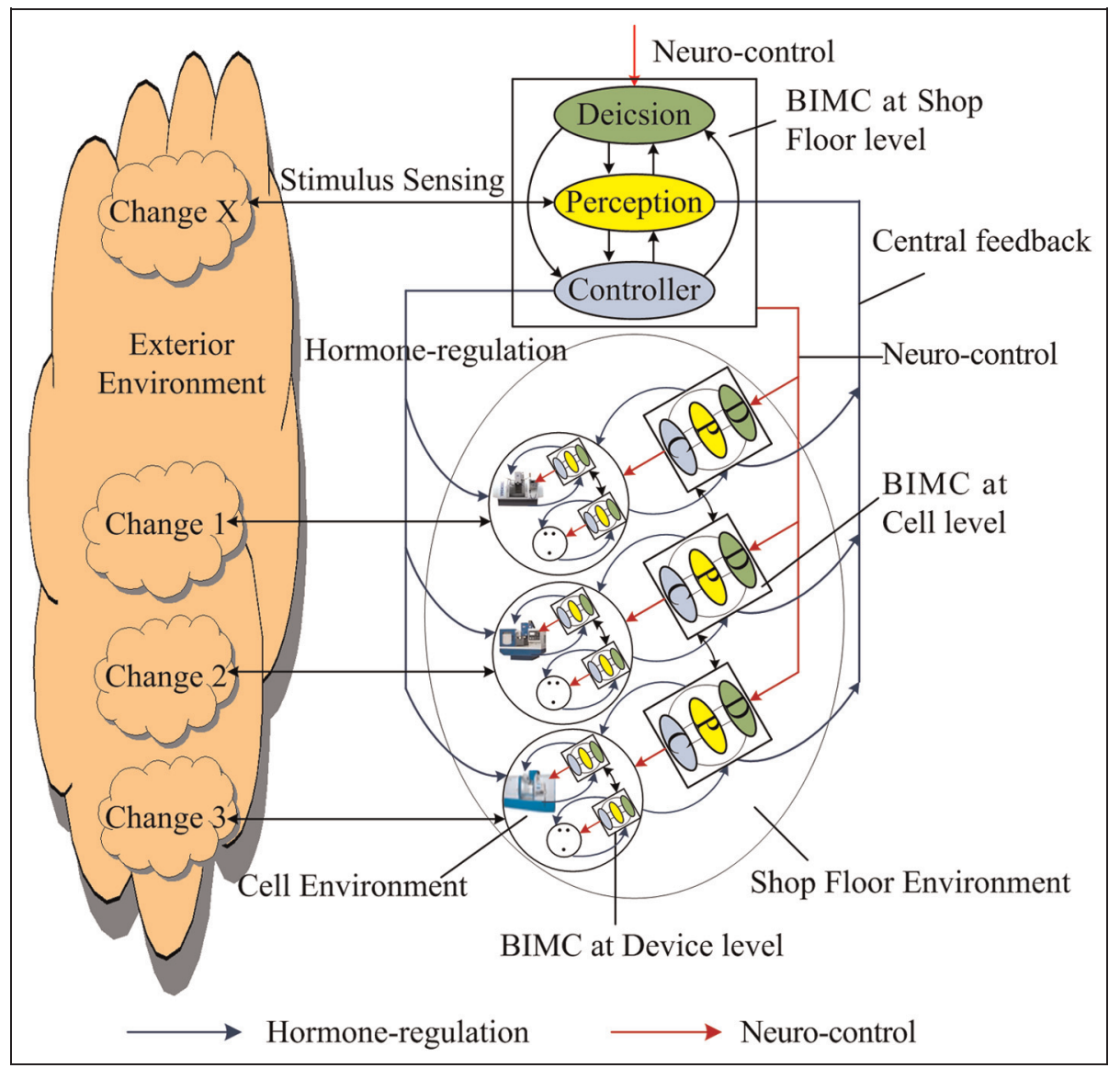

Figure I. NEIMS control architecture.

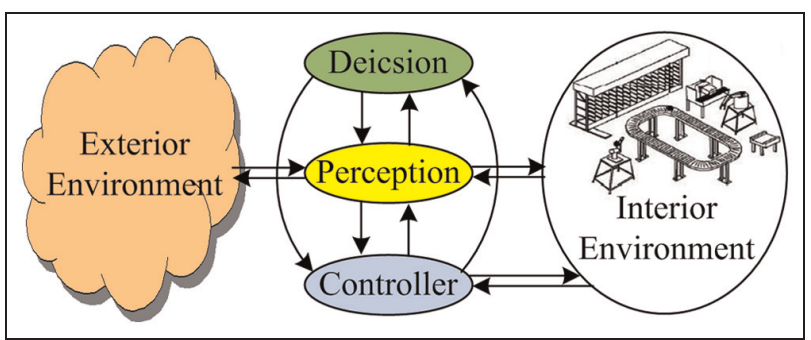

Figure 2. Basic structure of the BIMC.

and elaborate schedule plans due to its computing power and optimisation capability. The elaboration process only takes a short time because of the low complexity of the scheduling problems at this level. A schedule plan elaborated by a BIMC at the cell level is the optimal plan for the cell, but it is not the globally optimal plan because of a lack of global information.

A BIMC at the device level plays the role of performer to provide order execution services, and it can handle simple scheduling problems of its own based on its local knowledge.

\section{Dynamic re-scheduling model}

With respect to the NEIMS control architecture, a dynamic re-scheduling model is introduced in this section, which is the result of dynamic responses to stimuli between the BIMCs at different levels in the NEIMS.

At the operational level, the constraints of the manufacturing schedule are listed as follows:

1. One machine can process only one operation at a time.

2. One process cannot be started before its preceding processes are completed.

3. One BIMC has the capacity to execute a task $T_{j}=\left\{T_{l j}\right\}$, if $T Y P E_{j} \subseteq S_{i} \Leftrightarrow T Y P E_{l j} \in S_{i}$, where $S_{i}$ is the set of skills of BIMC $i, T Y P E_{j}=\left\{T Y P E_{l j}\right\}$ is the list of processing types of $T_{j}$, and $T Y P E_{l j}$ is the processing type of $T_{l j}$.

4. Pre-emption is not allowed in processing each job, that is, once an operation is started, it must be finished without interruption.

Figure 3 illustrates an example of how the dynamic re-scheduling works in NEIMS. In stage I (normal operation stage), that is, without the occurrence of unexpected events, the BIMCs at different levels are organised into a hierarchical structure, and the BIMC at the shop floor level elaborates and sends the globally optimised manufacturing schedule plans to the BIMCs at lower levels. The BIMCs at different levels follow 


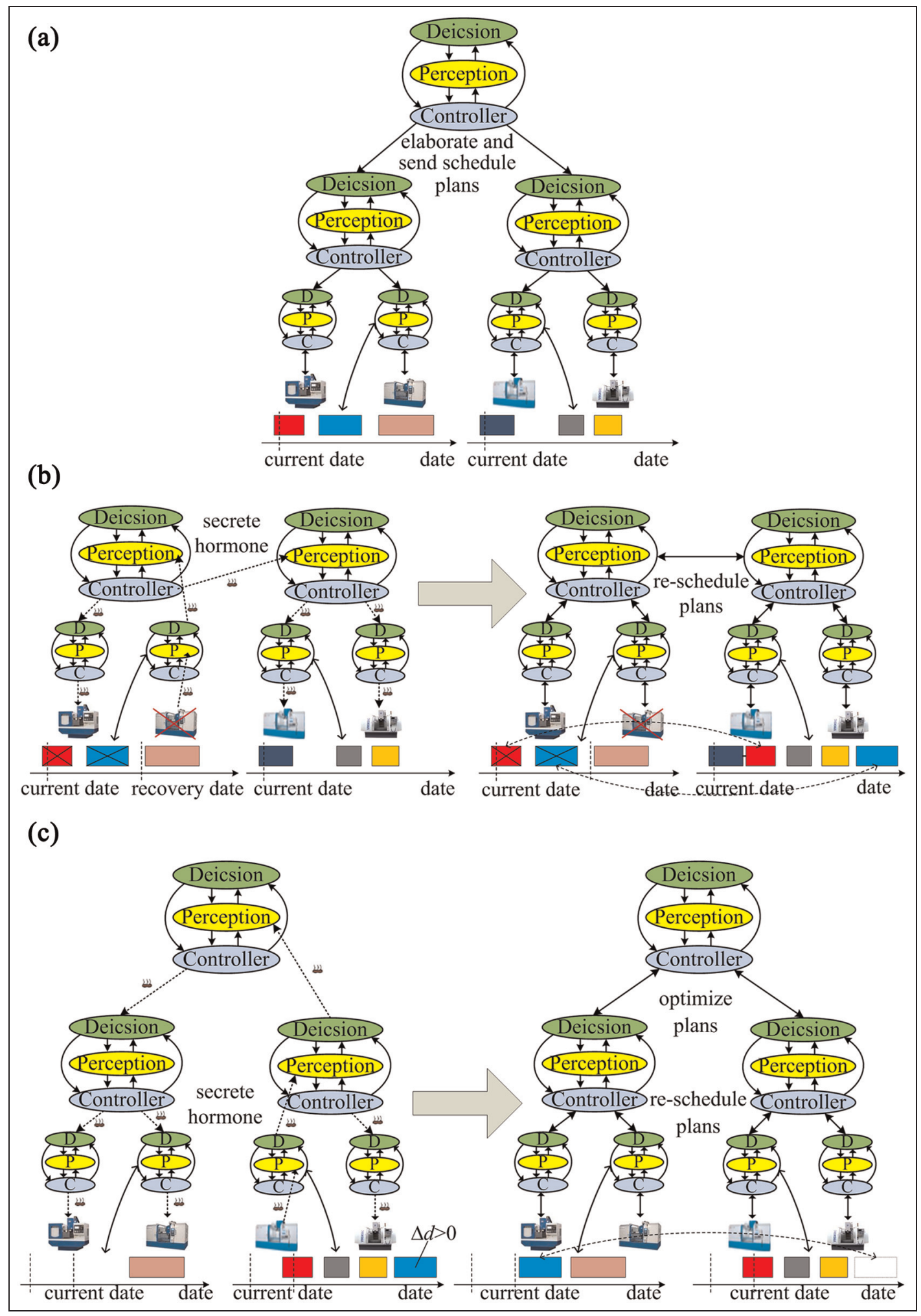

Figure 3. Dynamic re-scheduling in NEIMS: (a) normal operation stage, (b) response to disturbance stage, and (c) recovery after disturbance stage.

the received plans and perform their own stationary operations.

In NEIMS, reaction to the occurrence of unexpected events on the shop floor can be divided into two stages: response to the disturbance stage and recovery after the disturbance stage. In stage II (response to disturbance stage), when an unexpected disturbance occurs on the shop floor (e.g. machine failure), a hormone regulation mechanism adopted by NEIMS is triggered for system equilibrium. The hormone regulation processes in a body control the secretion of hormones to maintain a well-balanced biochemical environment in the vessels. 
For instance, if a body is suffering from a cold stimulus, the hypothalamus stimulates the pituitary and thyroid glands to secrete thyroxine to increase metabolism and maintain the body temperature at a stable level. Similarly, when certain task and resource deviations are detected in the relevant BIMC at the cell level, these events can be viewed as oscillations in the concentration of hormones. The deviations from the original plan stimulate the BIMC to cooperatively interact with other units to maintain the stability of the system. The related BIMCs will adjust their original plans from the device to the cell levels, and the BIMC that suffers the disturbance can enact an alternative schedule plan to ensure on-time product delivery while maintaining a low workin-process inventory without the supervision of the BIMC at the shop floor level. In the example illustrated in Figure 3(b), two tasks from a failed machine are allocated to another machine that provides similar skills.

In stage III (recovery after disturbance stage), after the execution of event-based re-scheduling for a period of time, the machine recovers from the malfunction. Certain tasks might be delayed from the planned due dates, and selected deviations from the due date can be detected in the BIMCs at the device level. In the case of such a context, a hormone regulation mechanism is adopted by NEIMS to adjust the system at balance. When it senses the deviation information, the BIMC at the shop floor level chooses certain bottleneck tasks according to the degree of the deviations from the due date and re-allocates these tasks through interaction among the BIMCs at different levels. If the delayed tasks are re-allocated, this means that the influence of a disturbance caused by a delay is solved or weakened, but if the delayed tasks cannot be re-allocated, the delay must be accepted by the relevant BIMC. As shown in the example illustrated in Figure 3(c), one task from a machine that will seriously deviate from the due date is allocated to another machine that provides a similar skill.

During the process of reaction to disturbances (from stage II to stage III), the BIMC at shop floor level does not directly address the disturbance but receives feedback from the interactions among the involved BIMCs, subsequently continues optimisation of the plan, and elaborates the task allocation for recovery.

\section{Resource allocation mechanism based on hormone regulation mechanism}

As mentioned previously, the dynamic re-scheduling model in NEIMS uses the hormone regulation mechanism to quickly react to disturbances in stages II and III. This section illustrates the resource allocation mechanism based on the hormone regulation mechanism.

Biological hormone regulation mechanism. A feedbackcontrolled ensemble model of the stress-responsive hypothalamus-pituitary-adrenal $\operatorname{axis}^{12}$ is used to explain the hormone regulation mechanism and the related control model. This neuroendocrine ensemble exhibits prominent time-dependent dynamics that are vividly reflected in the pulsatile and 24-h rhythmic output. Episodic secretion is driven by the hypothalamic neuronal pacemakers of the central nervous system (CNS), which secretes the pituitary signalling peptide $\mathrm{CRH}$ (adrenocorticotropic hormone (ACTH)-releasing hormone). These agonists synergistically stimulate ACTH synthesis and secretion, which in turn promotes the dose-responsive biosynthesis of cortisol. Cortisol feeds back to inhibit CRH and ACTH production via concentration-dependent and rapid rate-sensitive mechanisms. A typical control model of hormone regulation is shown in Figure 4, which illustrates the characteristic of negative feedback.

Hormone regulation model of NEIMS. Inspired by the biological hormone regulation mechanism, the hormone regulation model of NEIMS is proposed for coordination, as shown in Figure 5, and the notations of the model are listed as follows:

$G T$ : the global task of the original plan is a set of tasks that is elaborated and allocated by the BIMC at the shop floor level.

$G R$ : the global resource capacity of the original plan is a set of available resources on the shop floor.

$D D$ : the due date information refers to the $G T$.

PT: the planned task schedule for the relevant BIMC is a set of tasks allocated by the BIMC at the shop floor level.

$P R$ : the planned resource allocation to the relevant BIMC is a set of available resources that is used to execute $P T$.

$P D$ : the planned due date information refers to the $P T$

$T F$ : the task completed by the relevant BIMC is a set of tasks actually finished by the relevant BIMC.

$R C$ : the resource capacity consumed by the relevant BIMC is a set of resource capacities that is actually consumed by the relevant BIMC.

$A D$ : the actual due date information refers to the $T F$

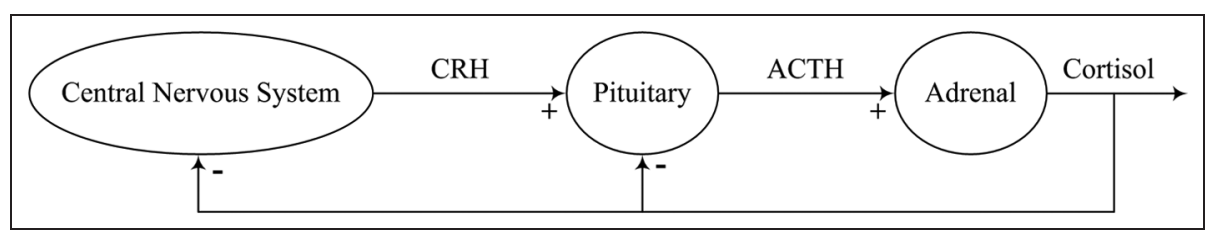

Figure 4. Negative feedback control model of hormone regulation. 


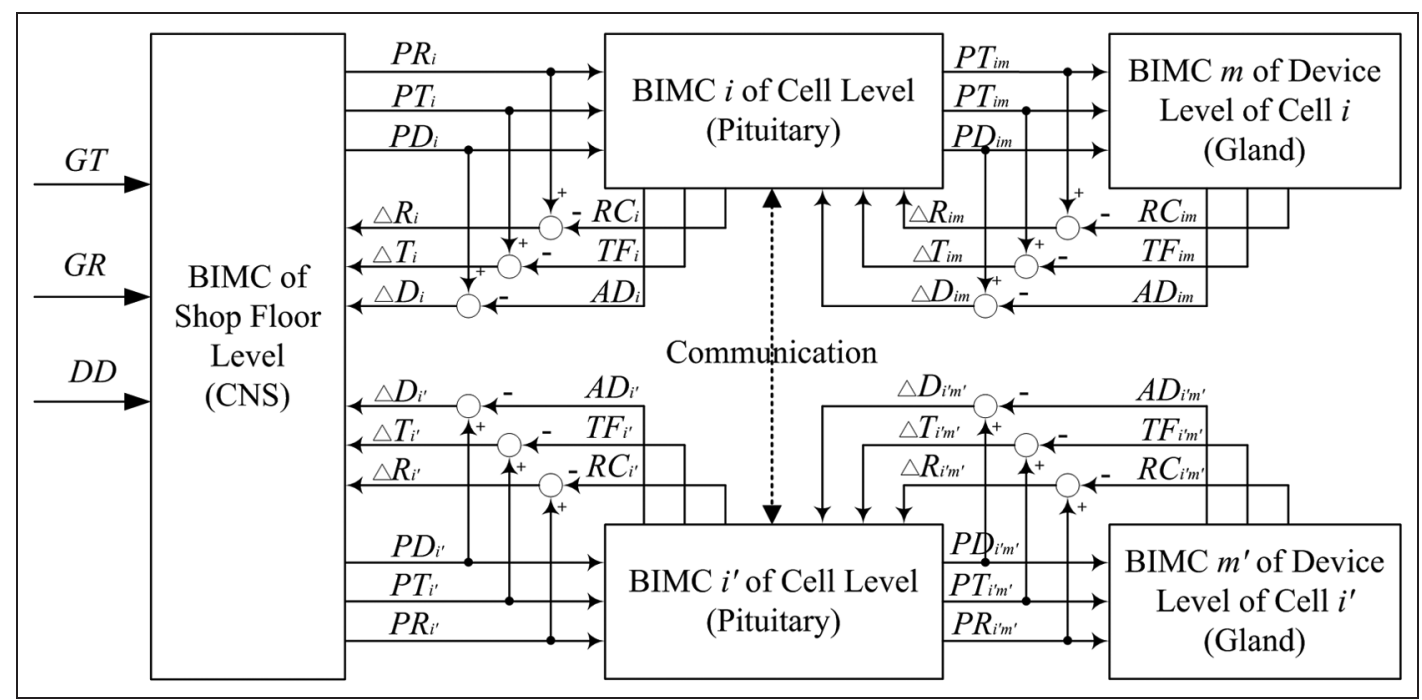

Figure 5. Hormone regulation model of NEIMS.

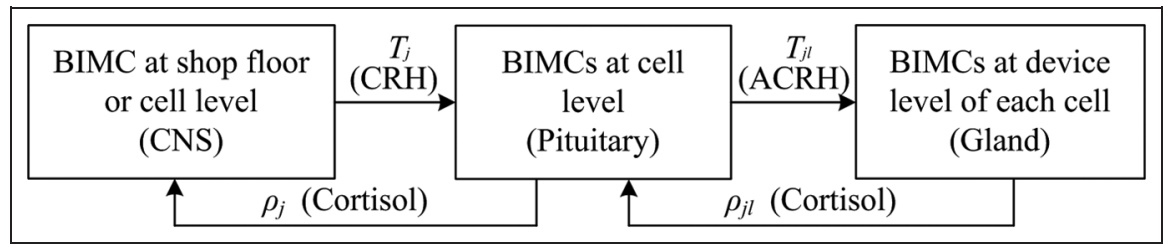

Figure 6. Dynamic responses to stimuli among BIMCs at different levels.

$\Delta R$ : the resource capacity deviation of the relevant BIMC is a set of resource capacities between $P R$ and $R C$.

$\Delta T$ : the task deviation of the relevant BIMC is a set of tasks between $P T$ and $T F$.

$\Delta D$ : the due date deviation of the relevant BIMC is a set of due date information between $P D$ and $A D$.

Based on the hormone regulation principle, the BIMCs at the shop floor level, cell level, and device level are separately treated as the CNS, pituitary, and the adrenal glands. Taking the deviations of the resources, tasks, and dates of delivery as three types of hormones, the levels of hormone concentrations of the BIMC can be indicated as $\Delta R, \Delta T$, and $\Delta D$. During the dynamic scheduling plan, the information for the resource capability deviation $\Delta R$ contains the machine type, capability, and number of machine units. The information for task deviation $\Delta T$ refers to the number, process route, and due date of the workpiece. The information of due date deviation $\Delta D$ includes the number of workpieces that cannot be completed on time, the number of machine units, and the deviation value of the due date. If a certain hormone concentration $(\Delta R, \Delta T$, or $\Delta D)$ is not equal to 0 , this means that unexpected events have occurred on the shop floor, and the hormone regulation processes will be triggered for maintenance of system equilibrium via resource regulation. Resource regulation is intended to re-organise the resource allocations to re-schedule and minimise deviations from the original plan. The details of the resource allocation mechanism for dynamic re-scheduling are detailed in the following section.

Resource allocation mechanism. Using the biological hormone regulation mechanism, the distributed resource allocation mechanism of NEIMS is proposed as shown in Figure 6, and the notations are given below:

$J$ is the set of jobs.

$L$ is the set of machining processes of a job.

$T_{j}(j \in J)$ is the task that can be performed by the BIMC at the cell level.

$T_{j l}(l \in L, j \in J)$ is the task that can be performed by the BIMC at the device level.

$\rho_{j}(j \in J)$ is the hormone price of performing the task $T_{j}$. $\rho_{j l}(l \in L, j \in J)$ is the hormone price of performing the task $T_{j l}$.

In the presence of deviations $(\Delta R, \Delta T$, and $\Delta D)$, a number of bottleneck tasks $\left(\left\{T_{j}\right\}\right.$, similar to $\left.\mathrm{CRH}\right)$ are selected and released into the shop floor environment by the BIMC at the shop floor or cell level. Stimulated by $T_{j}$, the BIMCs at the cell level segment $T_{j}$ into a set of tasks $\left(\left\{T_{j l}\right\}\right.$, similar to ACTH) that can be performed by machines and release them into each cell environment. Stimulated by $T_{j l}$, the BIMCs at the device level 
attempt to insert the new task into the original plans according to the priorities and determine the feasibility of performing those tasks by updating the hormone price ( $\rho_{j l}$ similar to cortisol). The BIMCs at cell or shop floor level evaluate the updated hormone prices and select an appropriate cell to assign the task. Hormone prices updated from the BIMCs at the device level are the prices (composed of the cost of performing the task and the influence on the resource utilisation rate) that must be paid for deviations from the original plan. The cost can be computed according to the following function

$$
C_{i m}^{T_{j l}}=P C_{i m}^{T_{j l}}+I C_{i m}^{T_{j l}}+S C_{i m}^{T_{j l}}
$$

where $P C_{i m}^{T_{j l}}$ is the processing cost, $I C_{i m}^{T_{j l}}$ is the inventory cost, and $S C_{i m}^{T_{j l}}$ is the shortage cost. The processing cost depends on the productivity of the relevant machine, and the inventory cost and shortage cost depend on the predicted deviation from the due date. The calculation process for the cost will not be described in this article.

Once the cost is calculated by the related BIMC at the device level, the influence on the resource utilisation rate must be considered, that is, a machine with a low cost but a high loading rate may lose to a machine with a slightly higher cost but a lower loading rate. To ensure that a machine with a low loading rate can be selected more easily, we propose a weight function for the loading rate inspired by the up- and downregulatory Hill functions ${ }^{13}$

$$
w_{i m}=\left\{\begin{array}{l}
\alpha\left(1+\frac{\left(L T-L R_{i m}\right)^{n_{c}}}{\left(H_{1}\right)^{n_{c}}+\left(L T-L R_{i m}\right)^{n_{c}}}\right), L R_{i m}<L T \\
\alpha\left(1-\frac{\left(L R_{i m}-L T\right)^{n_{c}}}{\left(H_{2}\right)^{n_{c}}+\left(L R_{i m}-L T\right)^{n_{c}}}\right), L R_{i m} \geqslant L T
\end{array}\right.
$$

where $L T$ is the appointed loading rate, $L R_{i m}$ is the actual loading rate of device $m$ in cell $i, H_{1}$ and $H_{2}$ are the respective thresholds of the up- and downregulatory Hill functions, $\alpha$ is a constant coefficient, $n_{c}$ is the Hill coefficient, and $n_{c} \geqslant 1$. The graph of the weighted function is shown in Figure 7, and the relevant parameters can be set as follows: $L T=60 \%$, $H_{1}=50, H_{2}=35, \alpha=1$, and $n_{c}=4$.

Based on the deviation between the appointed loading rate and the actual loading rate $\left(L T-L R_{i m}\right)$, the cost can be adjusted as follows. If $L T-L R_{i m}>20 \%$ (e.g. $L R_{\text {im }}=30 \%$ ), the cost must be decreased to ensure that the BIMC can be selected more easily. If $L T-L R_{i m}<-20 \%$ (e.g. $L R_{i m}=90 \%$ ), the cost will be raised to prevent the BIMC from being selected. If $L T-L R_{\text {im }} \in[-20 \%, 20 \%]$, the cost is maintained or finely adjusted, and the BIMC can be selected according to its actual cost. Inspired by equation (2) and the curve in Figure 7, the weighted cost of the hormone quantum considering the utilisation rate can be calculated as shown in equation (3)

$$
\rho_{i m}^{T_{j l}}=\frac{C_{i m}^{T_{j l}}}{w_{i m}}
$$

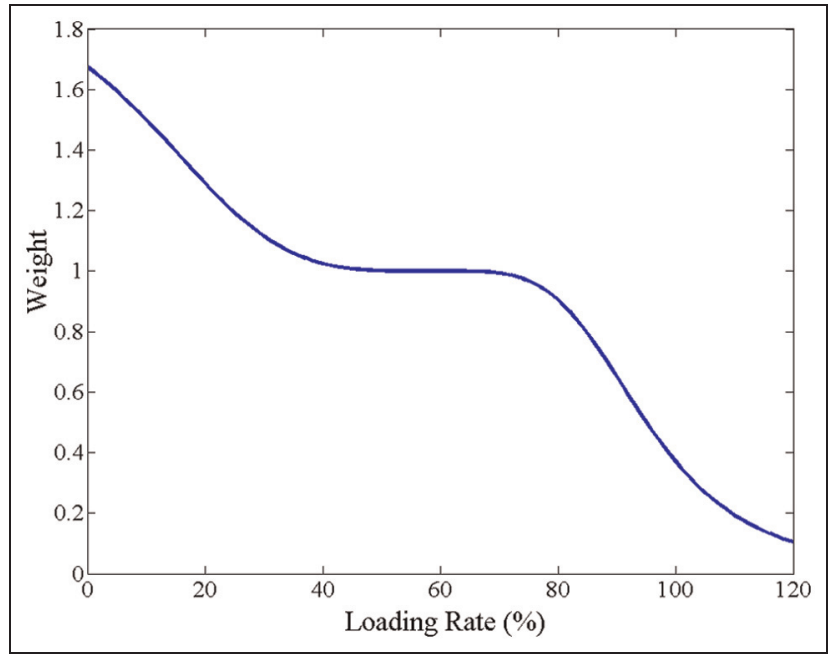

Figure 7. Curve of weight function of loading rate.

After calculating the hormone quantum of a device in the current state, the BIMC at the device level with the lowest hormone level will be selected by its cell. If the BIMC at the cell level senses several hormones with the same and lowest quantum from the BIMCs at the device level, it will select the BIMC with the lowest real loading rate.

In the process of resource allocation, the autonomous capability of each BIMC acts as the key concept, and the hormone regulation mechanism acts as the important function for support of the adaptive control and scheduling strategy. Those resources with lower cost can be detected from the bottom (device) level of the system, and at the same time, the resource utilisation rate can be balanced.

\section{Cooperation mechanisms for dynamic re- scheduling}

This section describes several types of dynamic rescheduling mechanisms triggered by unexpected disturbances.

\section{Dynamic re-scheduling for rush orders}

A rush order is an order (usually with high priority) that must be processed immediately after reaching the shop floor because it has an impending delivery time. These orders lead to a disturbance in the system because optimised manufacturing schedule plan was previously elaborated by the BIMC at the shop floor level. In this situation, the task deviations from that plan caused by rush orders act as the oscillation of task hormones and trigger the hormone regulation processes. As shown in Figure 8, the BIMCs at different levels follow the steps below to conduct their own inherent operations: 


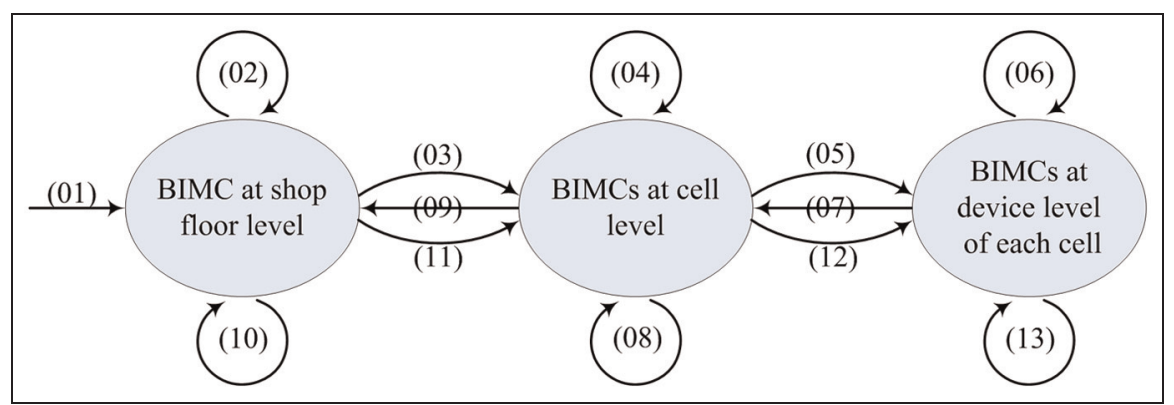

Figure 8. Dynamic re-scheduling caused by rush orders.

Step 01: when rush orders are accepted by the BIMC at the shop floor level, they $(\Delta G T)$ will be added to the global tasks.

Step 02: the BIMC at the shop floor level segments $\Delta G T$ into a set of tasks $\left\{\Delta T_{j}\right\}$ that can be performed by the cells.

Step 03: the BIMC at the shop floor level releases the task hormone information $\left(\Delta T_{j}\right)$ into the shop floor environment as CRH.

Step 04: when each BIMC at the cell level senses $\Delta T_{j}$, it will segment $\Delta T_{j}$ into a set of tasks $\left\{\Delta T_{j l}\right\}$ that can be performed by the machines.

Step 05: each BIMC at the cell level releases hormone information $\Delta T_{j l}$ into its relevant cell environment as ACTH.

Step 06: when the BIMC at the device level senses $\Delta T_{j l}$, it will abstract the processing type $\left(T Y P E_{l j}\right)$ from $\Delta T_{j l}$ and check its machine skills $\left(S_{i m}\right)$. If $T Y P E_{l j} \subseteq S_{i m}$, it will abstract the priority of the new task and attempt to insert the new task at the front of the lower priority tasks and re-schedule the original plan; in addition, it will evaluate the increase in the hormone quantum $\left(\rho_{i m}^{\Delta T_{j l}}\right)$ based on equation (3). If TYPE BIMC will do nothing. In the process of re-scheduling, the decision maker of the BIMC selects the plan $\left(p_{\text {im }}\right)$ from the set of alternative production plans that has the minimum increase in hormone quantum as the optimal plan.

Step 07: the BIMC at the device level feeds back the new plan with the increase in hormone quantum $\left(p_{i m}, \rho_{i m}^{\Delta T_{j l}}\right)$ to its cell as cortisol.

Step 08: when the BIMC at the cell level senses the feedback information $\left\{\left(p_{i m}, \rho_{i m}^{\Delta T_{j l}}\right)\right\}$ from the relevant devices, it will re-schedule the plan and select the optimal plan with the minimum increase of hormone quantum $\left(p_{i}, \rho_{i}^{\Delta T_{j}}\right)$ from the alternative production plans in the cell.

Step 09: the BIMC at the cell level feeds back the new plan with the increase of hormone quantum $\left(p_{i}, \rho_{i}^{\Delta T_{j}}\right)$ to the shop floor controller as cortisol.

Step 10: when the BIMC at the shop floor level senses the feedback information $\left\{\left(p_{i}, \rho_{i}^{\Delta T_{j}}\right)\right\}$, it will select the optimal plan with the minimum increase in hormone quantum $\left(p_{i}^{o p t}, \rho_{i}^{\Delta T_{j}}\right)$ from the alternative production plans.
Step 11: the BIMC at the shop floor level allocates $\Delta T_{j}$ to the relevant cell.

Step 12: the BIMC at the cell level allocates $\left\{\Delta T_{j l}\right\}$ to the relevant device(s).

Step 13: each BIMC at the device level executes the new plan.

\section{Dynamic re-scheduling for machine malfunction}

In the case of the occurrence of machine breakdown, task and resource deviations from plan as estimated by the relevant BIMC at the device level act as a type of hormone and trigger the hormone regulation process. As shown in Figure 9, the BIMCs at different levels follow the steps below to conduct their own inherent operations:

Step 01: the BIMC $m^{\prime}$ at the device level of the failed machine estimates the state of the machine, abstracts the task and resource hormone information $\left(\left\{\Delta T_{j l}\right\}\right.$ and $\left.\Delta R_{i^{\prime} m^{\prime}}\right)$, and updates this information to its cell. The $\left\{\Delta T_{j l}\right\}$ includes the information on the processes that cannot be performed during the downtime, and $\Delta R_{i^{\prime} m^{\prime}}$ includes the information on the state and downtime of the machine.

Step 02: the BIMC $i^{\prime}$ at the cell level of the failed machine releases $\Delta T_{j l}$ into the shop floor environment as CRH.

Step 03: when each BIMC at the cell level senses the hormone information $\left(\Delta T_{j l}\right)$, it will release $\Delta T_{j l}$ into its relevant cell environment as ACTH.

Step 04: when the BIMC at the device level senses $\Delta T_{j l}$, it will abstract the processing type $\left(T Y P E_{l j}\right)$ from $\Delta T_{j l}$ and check the machine skills $S_{i m}$. If $T Y P E_{l j} \subseteq S_{i m}$, the BIMC will attempt to insert the new task into the original plan and re-schedule and will subsequently evaluate the increase in hormone quantum $\left(\rho_{i m}^{\Delta T_{j l}}\right)$ based on equation (3); if $T Y P E_{l j} \not \subset S_{i m}$, it will do nothing. In the process of re-scheduling, the decision maker of the BIMC selects the plan $\left(p_{i m}\right)$ with the minimum increase in hormone quantum from the alternative production plans as the optimal plan.

Step 05: the BIMC at the device level feeds back the new plan with the increase in hormone quantum $\left(p_{i m}, \rho_{i m}^{\Delta T_{j l}}\right)$ to its cell as cortisol. 


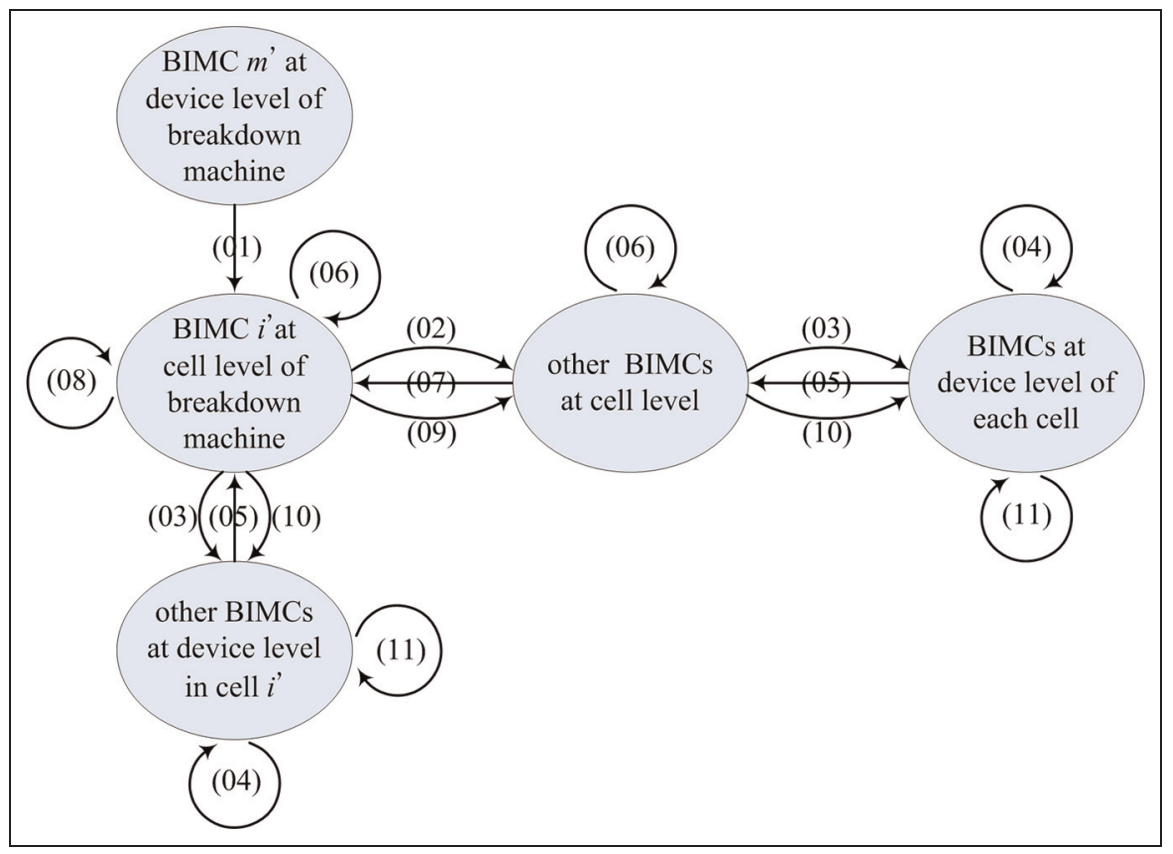

Figure 9. Dynamic re-scheduling caused by machine malfunction.

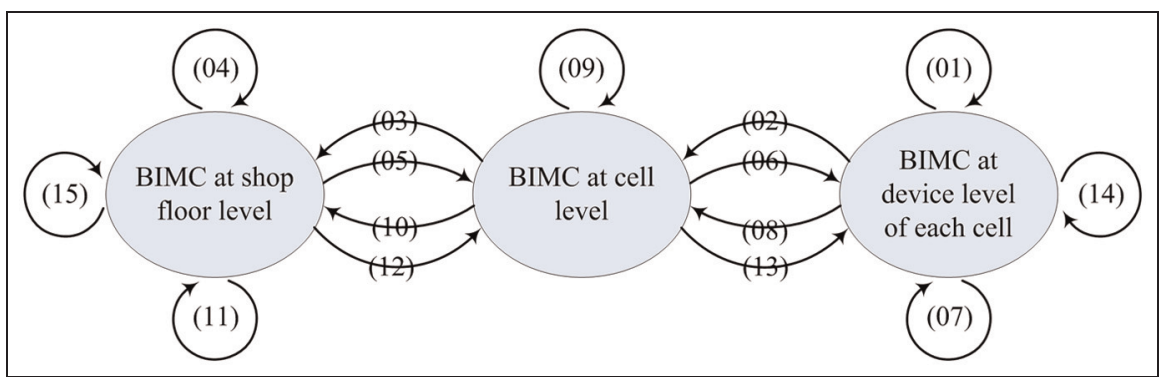

Figure 10. Dynamic scheduling caused by delays.

Step 06: when the BIMC at the cell level senses the feedback information $\left\{\left(p_{i m}, \rho_{i m}^{\Delta T_{j l}}\right)\right\}$ from the relevant BIMCs at the device level, it will re-schedule the plan and select the optimal plan with the minimum increase of hormone quantum $\left(p_{i}, \rho_{i m}^{\Delta T_{j l}}\right)$ from the alternative production plans in the cell.

Step 07: the BIMC at the cell level feeds back the new plan with the increase in hormone quantum $\left(p_{i}, \rho_{i m}^{\Delta T_{j l}}\right)$ to the BIMC $i^{\prime}$ cell level as cortisol.

Step 08: when the BIMC $i^{\prime}$ at the cell level senses the feedback information $\left\{\left(p_{i}, \rho_{i m}^{\Delta T_{j l}}\right)\right\}$, it will select the optimal plan $\left(p_{i}^{o p t}, \rho_{i m}^{\Delta T_{j l}}\right)$ with the minimum increase in hormone quantum from the alternative production plans.

Step 09: the BIMC $i^{\prime}$ at the cell level allocates $\Delta T_{j l}$ to the relevant cell.

Step 10: the BIMC at the cell level allocates $\Delta T_{j l}$ to the relevant device(s).

Step 11: each BIMC at the device level executes the new plan.

\section{Dynamic re-scheduling for delays}

An operational delay can occur after disturbances if the BIMCs at the device level detect due date deviations. In this situation, the task deviations from plan caused by the delays act as a type of hormone and trigger the hormone regulation process. As shown in Figure 10, the BIMCs at different levels follow the steps below to conduct their own inherent operations:

Step 01: the BIMC at the device level affected by the unexpected events detects the processing state of the machine and abstracts task deviations $\left(\Delta T_{j l}, \Delta d_{j l}^{\Delta T_{j l}}\right.$, $\left.\rho_{\text {im, orig }}^{\Delta T_{j l}}\right)$ with the due date deviations and increases in hormone quantum of the relevant tasks.

Step 02: the BIMC at the device level updates $\left(\Delta T_{j l}, \Delta d_{j l}^{\Delta T_{j l}}, \rho_{\text {im, orig }}^{\Delta T_{j l}}\right)$ to its own cell.

Step 03: when the BIMC at the cell level senses the information $\left\{\left(\Delta T_{j l}, \Delta d_{j l}^{\Delta T_{j l}}, \rho_{\text {im, orig }}^{\Delta T_{j l}}\right)\right\}$ from its devices, it will collect and update them to the BIMC at the shop floor level. 
Step 04: after collecting the information from all cells, the BIMC at the shop floor level chooses several bottleneck tasks $\left\{\Delta T_{j l}\right\}$ for which there are serious deviations from the due date.

Step 05: the BIMC at the shop floor level releases the task hormone information $\Delta T_{j l}$ into shop floor environment as CRH.

Step 06: when each BIMC at the cell level senses $\Delta T_{i j}$, it will directly release $\Delta T_{i j}$ into its relevant cell environment as ACTH.

Step 07: when the BIMC at the device level senses $\Delta T_{j l}$, it will abstract the processing type $\left(T Y P E_{l j}\right)$ from $\Delta T_{j l}$ and check the machine skills $S_{i m}$. If $T Y P E_{l j} \subseteq S_{i m}$, it will attempt to insert a new task into the original plan and re-schedule and subsequently evaluate the increase in hormone quantum $\left(\rho_{i m}^{\Delta T_{j l}}\right)$ based on equation (3); if $T Y P E_{l j} \not \subset S_{i m}$, it will do nothing. In the process of rescheduling, the decision maker of the BIMC selects the plan $\left(p_{i m}\right)$ with the minimum increase in hormone quantum from the alternative production plans as the optimal plan.

Step 08: the BIMC at the device level feeds back the new plan with the increase in hormone quantum $\left(p_{i m}, \rho_{i m}^{\Delta T_{j l}}\right)$ to its cell as cortisol.

Step 09: when the BIMC at cell level senses the feedback information $\left\{\left(p_{i m}, \rho_{i m}^{\Delta T_{j l}}\right)\right\}$ from the relevant devices, it will re-schedule the plan and select the optimal plan $\left(p_{i}, \rho_{i m}^{\Delta T_{j l}}\right)$ with the minimum increase in hormone quantum from the alternative production plans in the cell.

Step 10: the BIMC at the cell level feeds back the new plan with the increase of hormone quantum $\left(p_{i}, \rho_{i m}^{\Delta T_{j l}}\right)$ as cortisol to the BIMC at the shop floor level.

Step 11: when the BIMC at the shop floor level senses the feedback information $\left\{\left(p_{i}, \rho_{i m}^{\Delta T_{j l}}\right)\right\}$, it will select the optimal plan $\left(p_{i}^{o p t}, \rho_{i m}^{\Delta T_{j l}}\right)$ with the minimum increase in hormone quantum from the alternative production plans. If $\rho_{i m}^{\Delta T_{j l}}<\rho_{i m, o r i g}^{\Delta T_{j l}}$, the new plan of $\Delta T_{i j}$ is better, and go to Step 12; if $\rho_{i m}^{\Delta T_{j l}} \geqslant \rho_{i m, o r i g}^{\Delta T_{j l}}$, the original plan of $\Delta T_{i j}$ is better, and go to Step 15 ;

Step 12: the BIMC at the shop floor level allocates $\Delta T_{i j}$ to the relevant cell.

Step 13: the BIMC at the cell level allocates $\Delta T_{j l}$ to the relevant device(s).

Step 14: the relevant BIMC at the device level executes the new plan. If tasks remain with due date deviations, the BIMC abstracts the task information and goes to Step 02.

Step 15: ending all the allocations of $\left\{\Delta T_{i j}\right\}$, the BIMC at the shop floor level begins global optimisation of the plan.

\section{Test bed for NEIMS simulation}

A test bed (shown in Figure 11) was set up to simulate the dynamic re-scheduling approach of NEIMS. An Advanced RISC Machine (ARM) controller linked with a Pentium IV personal computer serves as the
BIMC controller at the shop floor level and is responsible for the role of the CNS. Several ARM controllers act as the BIMC controllers at the cell level and are linked through a CAN bus to communicate with each other without the host computer. Wireless communication technology is adopted between the ARM controllers and several auto-guided vehicles (AGVs). Several single chips act as the BIMC controllers at the device level. One automated storage/retrieval system (AS/RS) was set up to store the raw materials and machined parts. A liquid-crystal display (LCD) thin film transistor (TFT) screen at the shop floor level displays the real-time operation situation of the entire system, and several LCD TFT screens at the cell level display the machine status of the cells. The physical test bed that simulates the operation of the entire system is shown in Figure 12.

\section{Simulation study}

To obtain benchmarking for dynamic re-scheduling approach implementation in NEIMS, the simulation experiments were performed over several different models with consideration of different disturbance conditions according to the framework defined by Cavalieri et al. ${ }^{14}$ In the experiment, no set-up is required because each machine is equipped with the tools required to execute a range of operations, and work orders are executed in the exact estimated manufacturing time. The experimental case study considers four types of orders with different plant loads. Each individual order includes four to six jobs, and each job is composed of two to four machining processes. Different orders arrive sequentially at the NEIMS, and jobs that belong to the same order arrive in the system at the same time. In the experiment, three plant scenarios ${ }^{15}$ are considered:

1. No unexpected disturbances occur.

2. Occurrence of failure in one turning machine of BIMC 1 at the cell level with a probability of $20 \%$.

3. Occurrence of failures in two turning machines of BIMC 1 and BIMC 2 at the cell level with a probability of $20 \%$.

\section{Performance indicators}

In the manufacturing context, the performance indicators can be classified as qualitative or quantitative. In this study, the quantitative indicators used to evaluate the implementation of dynamic re-scheduling approach in NEIMS are output rate, resource utilisation, leadtime, and lateness.

The output rate is an indicator of the productivity of a manufacturing system and is defined as the ratio between the output and the duration of the reference period. The resource utilisation is defined as the percentage of processing time during a time interval. The lead-time is the total time required to process a 


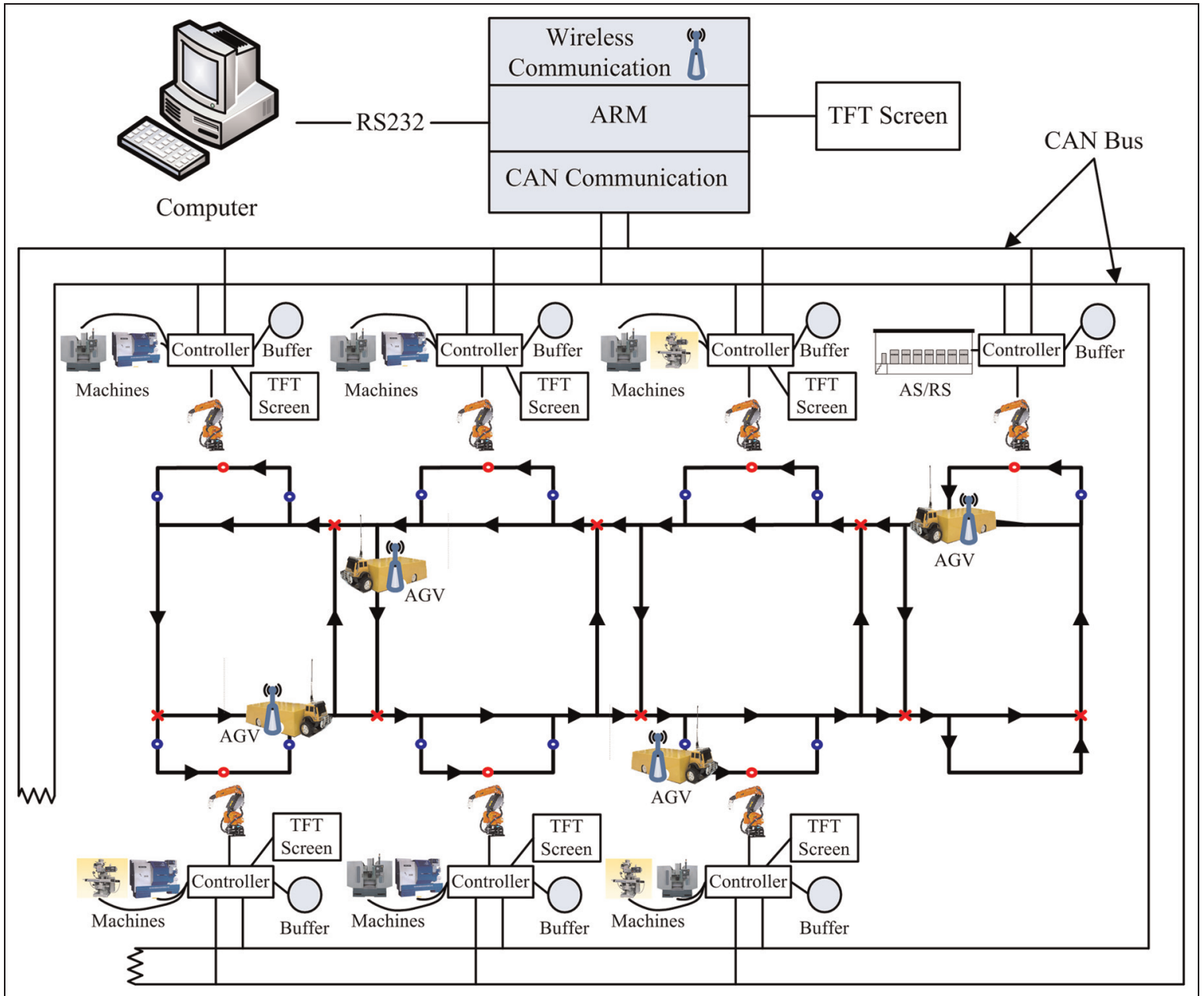

Figure I I. Simulation structure of NEIMS.

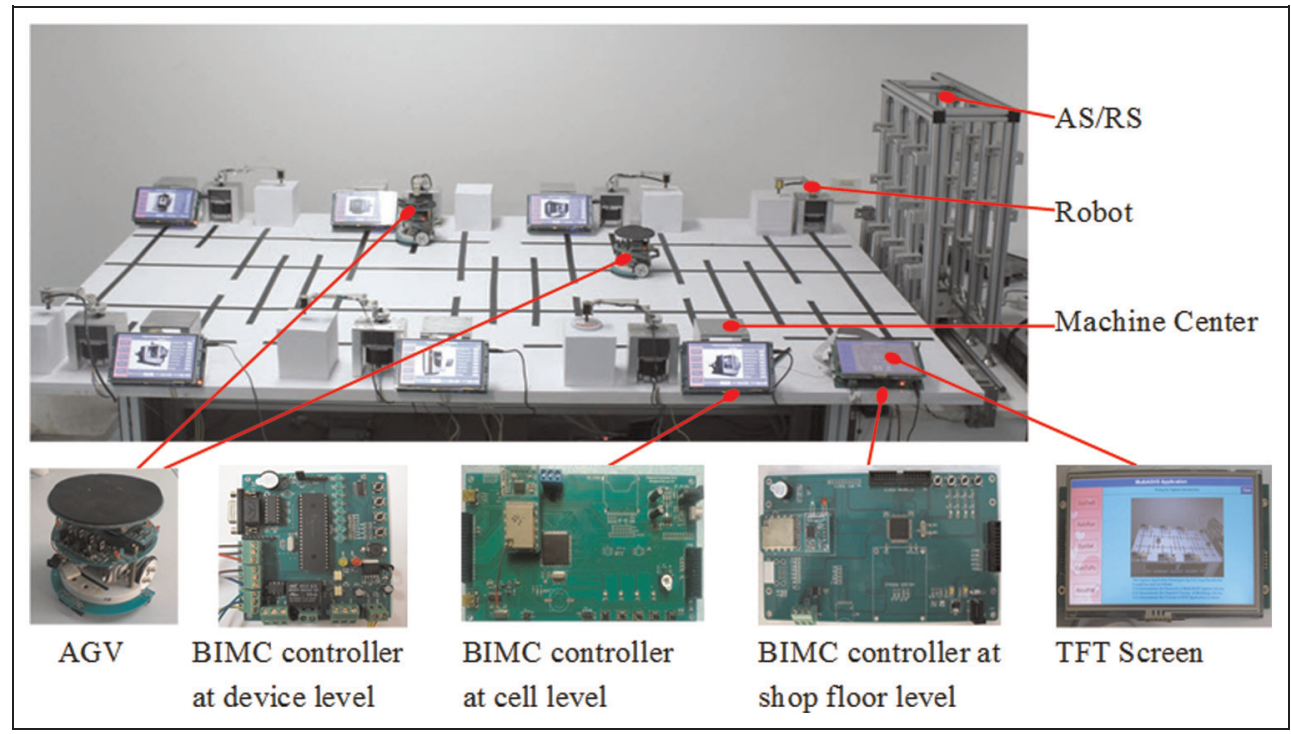

Figure I 2. Physical test bed of NEIMS.

given product through the factory plant and includes the post-process waiting time, the transport time, the pre-process waiting time, the set-up time, and the processing time. The shorter the lead-time, the more products can be produced by the production plant in the same period of time. The lateness is the deviation between the actual processing end and the target process end. 
In this study, the two qualitative indicators defined and tested to analyse the performance in NEIMS are robustness and agility.

The robustness is the ability of a manufacturing system to continue working correctly and relatively stably in the face of internal and external unexpected disturbances. ${ }^{16}$ In this article, the robustness is measured by introducing possible disturbances and verifying whether the manufacturing system continues to work correctly. For example, the system quickly reacts to the occurrences of machine breakdown, new rush orders, increases in the number of orders, and so on.

The agility of a manufacturing system is its ability to rapidly react to the occurrence of unexpected disturbances. ${ }^{17}$ In this article, the agility of a manufacturing system is evaluated using the loss of output rate

$$
\text { lopr }=\left(1-\frac{o p r_{\text {transient }}}{o p r_{\text {steady }}}\right) \times 100 \%
$$

where $o p r_{\text {steady }}$ is the output rate in a steady scenario and opr transient is the output rate in a transient scenario after the occurrence of disturbance. The loss of output rate directly reflects the agility of a manufacturing system. The smaller the lopr, the higher the agility of the manufacturing system will be.

Using simulation of the proposed approach in the test bed, various scales can be constructed to evaluate the performance of NEIMS.

\section{Analysis of results}

To compare the dynamic re-scheduling approach implemented in NEIMS, the same manufacturing system is modelled with the conventional control architecture. The conventional control approach is a traditional centralised control system that uses the centralised controller as the shop floor controller. Both the scheduling approaches use the same test bed:

1. In the conventional approach, the orders are scheduled according to the earliest due date first (EDD) rule, and the parts flow along the processes according to the first-in-first-out (FIFO) rule. If the disturbances are not addressed on the shop floor, the shop floor controller must optimise the schedules of every machine and attempt to react to the disturbances.

2. In the NEIMS approach, the BIMCs at different levels are organised in a hierarchical structure, and the shop floor controller elaborates and sends schedule plans to the cell controllers of BIMCs in the normal state. The NEIMS adopts the hormone regulation mechanism to agilely adjust its behaviours for recovery in the disturbance state.

Figure 13 illustrates four quantitative indicators that are extracted from the experiments simulated in the test bed. The result shows that the NEIMS approach produces higher values for output rate and resource utilisation and lower values for lead-time and lateness than the conventional approach, thus reflecting better production optimisation.

The robustness results of the two approaches are illustrated as follows. In the conventional approach, the shop floor controller optimises the schedules of the entire system at the time of machine breakdown and



Figure 13. Quantitative comparison results. 


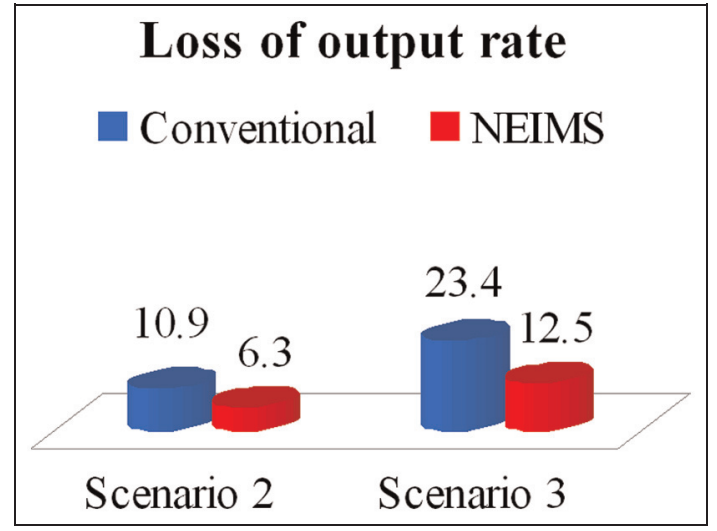

Figure 14. Loss of output rate comparison results.

recovery. The system must wait until the new schedules are calculated. In the NEIMS approach, the system can dynamically respond to the machine breakdown and recovery and maintains stability without breakdown of the entire system. The result shows that the NEIMS approach produces higher robustness than the conventional approach.

Figure 14 illustrates the loss of output rate of the evaluated approaches. The result shows that NEIMS approach results in lower values of loss of output rate than the conventional approach, thus reflecting a higher agility.

Analysing the qualitative and quantitative indicators simultaneously, the experimental results reveal that the proposed approach has the potential to improve the system performance.

\section{Conclusion}

In this article, we presented a dynamic shop floor rescheduling approach in NEIMS. The dynamic rescheduling approach, which is inspired by hormone regulation, is used to agilely respond to uncertain disturbances and maintain global optimisation and system stability. The computing power and optimisation capabilities embedded in the BIMCs at different levels play important roles in realising fast dynamic re-scheduling in NEIMS. The influences of delivery time and loading rate considered in the price hormone calculations ensure that lateness and resource utilisation can be solved and balanced at the bottom level. A test bed was built to simulate and verify the proposed dynamic rescheduling approach in NEIMS. Finally, a simulation case study was conducted to analyse the qualitative and quantitative indicators. The experimental results prove that the NEIMS approach combines good global production optimisation, strong system robustness, and high system agility.

The aim of this research primarily focuses on rapid and dynamic re-scheduling in NEIMS to address unexpected disturbances on the shop floor. During the process of re-scheduling, several manufacturing constraints (e.g. energy efficiency and human resources) were not taken into consideration. Therefore, future work should be directed towards application of the dynamic re-scheduling approach in more complex and practical manufacturing contexts to prove its extensive practicability. This article presents a contrast between the two approaches for potential to improve the system performance. However, the contrast between the proposed approach and other dynamic re-scheduling approaches has not been implemented. Hence, in future work, several typical dynamic re-scheduling approaches should be taken into consideration in the case design to verify the advantages and disadvantages of various approaches.

\section{Declaration of conflicting interests}

The authors declare that no conflict of interest exists.

\section{Funding}

This research was sponsored by the National Natural Science Foundation of China (NSFC) under Grant No. 51175262 and No. 61105114 and the Jiangsu Province Science Foundation for Excellent Youths under Grant BK20121011. This research was also sponsored by the CASES project supported by a Marie Curie International Research Staff Exchange Scheme Fellowship within the 7th European Community Framework Programme under grant agreement No. 294931.

\section{References}

1. Maravelia CT and Sung C. Integration of production planning and scheduling: overview, challenges and opportunities. Comput Chem Eng 2009; 33(12): 19191930.

2. Yandra and Hiroyuki T. A new multiobjective genetic algorithm with heterogeneous population for solving flowshop scheduling problems. Int J Comp Integ M 2007; 20(5): 465-477.

3. Fattahi $\mathrm{P}$ and Fallahi A. Dynamic scheduling in flexible job shop systems by considering simultaneously efficiency and stability. CIRP J Manuf Sci Technol 2010; 2(2): 114-123.

4. Rakesh KP, Ajai J and Rajiv V. An approach for integration of process planning and scheduling. Int $J$ Comp Integ $M$ 2003; 26(4): 282-302.

5. Renna P. Multi-agent based scheduling in manufacturing cells in a dynamic environment. Int J Prod Res 2011; 49(5): 1285-1301.

6. Qin L and Kan S. Production dynamic scheduling method based on improved contract net of multi-agent. In $\mathrm{Du} \mathrm{Z}$ (ed.) Intelligence computation and evolutionary computation. Berlin, Heidelberg: Springer, 2013, pp.929936.

7. Iwamura K, Mayumi N, Tanimizu Y, et al. A study on real-time scheduling for holonic manufacturing systems application of reinforcement learning. In Shirase K and Aoyagi S (eds) Service robotics and mechatronics. London: Springer, 2010, pp.201-204. 
8. Jana KT, Bairagi B, Paul S, et al. Dynamic schedule execution in an agent based holonic manufacturing system. J Manuf Syst 2013; 32(4): 801-816.

9. Dan ZG, Cai LN and Zheng L. Improved multi-agent system for the vehicle routing problem with time windows. Tsinghua Sci Technol 2009; 14(3): 407-412.

10. Hsieh FS. Developing cooperation mechanism for multiagent systems with Petri nets. Eng Appl Artif Intel 2009; 22(4-5): 616-627.

11. Tang $\mathrm{D}, \mathrm{Gu} \mathrm{W}$, Wang $\mathrm{L}$, et al. A neuroendocrineinspired approach for adaptive manufacturing system control. Int J Prod Res 2011; 49(5): 1255-1268.

12. Keenan DM, Licinio J and Veldhuis JD. A feedbackcontrolled ensemble model of the stress-responsive hypothalamo-pituitary-adrenal axis. $P$ Natl Acad Sci USA 2001; 98(7): 4028-4033.
13. Farhy LS. Modeling of oscillations of endocrine networks with feedback. Method Enzymol 2004; 384: 54-81.

14. Cavalieri S, Macchi M and Valckenaers P. Benchmarking the performance of manufacturing control systems: design principles for a web-based simulated test bed. $J$ Intell Manuf 2003; 14(1): 43-58.

15. Leitao P and Restivo F. A holonic approach to dynamic manufacturing scheduling. Robot Cim: Int Manuf 2008; 24(5): 625-643.

16. Bal M and Hashemipour M. Virtual factory approach for implementation of holonic control in industrial applications: a case study in die-casting industry. Robot Cim: Int Manuf 2009; 25(3): 570-581.

17. Leitao P. An agile and adaptive holonic architecture for manufacturing control. PhD Thesis, University of Porto, Porto, 2004. 